\title{
Evaluation of prediction models applied to the soil-water characteristic curve of ideal materials
}

\author{
Alves Roberto Dutra ${ }^{1 *}$, Gitirana Jr. Gilson de F. N. ${ }^{1}$, Vanapalli Sai K. ${ }^{2}$ \\ ${ }^{1}$ Universidade Federal de Goiás, Goiânia, Goiás, Brazil \\ ${ }^{2}$ University of Ottawa, Ottawa, Ontario, Canada
}

\begin{abstract}
The development of theoretical and semi-empirical models to study capillary mechanisms and predict the soil-water characteristic curve (SWCC) generally requires the idealization of pore space and pore water, considering simplifying hypotheses. The study of ideal materials comprised of particles with controlled shape and size allows the evaluation of such simplifying hypotheses and the subsequent generalization to actual soils. In this paper, four theoretical and semi-empirical models for the prediction of the SWCC are applied to the prediction of artificial materials comprised of spherical particles. Nineteen grain-size distribution curves, with varying coefficients of uniformity are considered. The dataset is comprised of materials previously published and additional tests carried out by the authors, under highly controlled conditions. The analyses allowed the evaluation of the effect of grain-size distribution curve and shape of the particles. The limitations and advantages of each prediction model was investigated, and a detailed comparison is presented, guiding future implementations of improved models.
\end{abstract}

\section{Introduction}

The soil-water characteristic curve (SWCC) has proved to provide some of the most fundamental and relevant information regarding unsaturated soils' behaviour. For unsaturated soils, the SWCC is as relevant as the grain-size distribution is for saturated soils. Several analyses, behaviour predictions, and properties can be estimated based on SWCC, which make it the key to the implementation of unsaturated soils mechanics. The SWCC is influenced by several retention mechanisms, with capillary retention being the most important. Therefore, the SWCC is influenced by soil mineralogy, grain and pore-size distribution, porosity, surface tension, texture, fabric, particle shape, wetting-drying cycles, the contact angle, entrapped air, among others factors.

The complex retention behaviour of soils has led many researches to develop models to estimate the SWCC using relatively simple properties, such as the grain-size distribution (GSD) and void ratio $(e)$. The works of [1], [5], [2], [6], [7], [3], [8] and [4] are few examples of these methods; however, each model is based on different sets of hypotheses and, therefore, has limitations, providing unreliable results and being applicable to a limited range of soils.

This paper presents a comparison of the performance of the prediction models proposed by [1-4], using glass beads. These artificial materials are nearly spherical, have relatively smooth surface, and present low variability of their properties, making them some of the simplest material available to evaluate the performance of prediction models.

\section{Description of the prediction models}

\subsection{Arya and Paris [1] and Arya and Dierolf [2]}

Arya and Paris [1], observing the similarities between the GSD and the SWCC, developed a model that transforms the grain-size distribution curve into a poresize distribution (PSD). This model is based on the following simplified hypotheses:

- The bulk density is the same for all fractions;

- The particles of each fraction are assumed spherical with diameter equals to the mean diameter of the fraction;

- The pore volume of each fraction is approximated to the volume of a capillary cylinder with its radius associated to the mean radius of the particles; and

- Hysteresis is neglected.

The first step is to divide the GSD curve in $n$ fractions, each one with a different mean pore diameter. For each fraction, the volume of voids $\left(V_{v i}\right)$ is calculated as:

$$
V_{v i}=\frac{m_{s i}}{\rho_{s}} e
$$

where: $i=1,2,3, \ldots, n ; m_{s i}$ is the mass of the $i^{\text {th }}$ fraction; $\rho_{s}$ is the density of the particles; and $e$ is the 
void rate. Also, it is considered that the sum of $m_{s i}$ is equal to one, which makes the value of $m_{s i}$ equals to its respective percent passing (not accumulated), as represented by the GSD curve.

The accumulated volumetric water content $\left(\theta_{v i}\right)$ stored up to the fraction $\mathrm{i}$ is given by:

$$
\theta_{v i}=\sum_{i=1}^{n} \frac{V_{v i}}{V}=\sum_{i=1}^{n} V_{v i} \rho_{d r y}
$$

where: $V$ is the total volume of the soil and $\rho_{d r y}$ is the dry density.

For a given particle size range, a mean volumetric water content corresponding to its midpoint can be calculated by:

$$
\theta_{v i}^{*}=\frac{\theta_{v i}+\theta_{v i+1}}{2}
$$

Because the authors assumed the particles having a spherical shape and the voids forming a capillary tube, it is possible to describe the particles and volume of voids as:

$$
\begin{gathered}
V_{s i}=\frac{n_{i} 4 \pi R_{i}^{3}}{3}=\frac{m_{s i}}{\rho_{s}} \\
V_{v i}=\pi r_{i}^{2} h_{i}=\frac{m_{s i}}{\rho_{s}} e
\end{gathered}
$$

where for the particles of the $i^{\text {th }}$ fraction, $V_{s i}$ is its volume; $n_{i}$ is the number of particles; $R_{i}$ is the radius the particles. The radius of the cylindric capillary tube is $r_{i}$ and $h_{i}$ is the total pore length, for the $i^{\text {th }}$ fraction.

Considering the soil having a cubic packing assemblage, the total pore length $\left(h_{i}\right)$ is given by $n_{i} 2 R_{i}$. However, the authors suggest the adoption of an empirical parameter $(\alpha)$ in order to consider the nonspherical shape of the particles. Therefore, the total pore length $\left(h_{i}\right)$ becomes $n_{i}^{\alpha} 2 R_{i}$. Combining the pore length with Equation 5, results in the following equation for $r_{i}$ :

$$
r_{i}=R_{i} \sqrt{\frac{4 e n_{i}^{1-\alpha}}{6}}
$$

Finally, matric suction can be estimated using the Laplace's equation and assuming that the water menisci are spherical caps with a single radius of curvature:

$$
\left(u_{a}-u_{w}\right)=\frac{2 T_{s}}{r_{i}}
$$

where: $u_{a}$ is the pore-air pressure; and $u_{w}$ is the porewater pressure, and $T_{s}$ is the surface tension.

The parameter $\alpha$ was evaluated by [1] for several soils, with different textures. The authors recommended the adoption of values of $\alpha$ varying from 1.31 to 1.43 .
Comparing the predicted curve to laboratory data, reasonable agreement was observed. The overall shape of the SWCC was adequately represented, indicating the strong relationship between the PSD curve estimated from the GSD and the SWCC. The effect of $\alpha$ in the predicted curve was mainly in terms of its overall position. Changes in $\alpha$ produce a translation of the entire SWCC along the matric suction axis. Therefore, it is reasonable to conclude that the empiricism introduced by the $\alpha$ parameter is necessary because the simplifying hypotheses that produced an unrealistic measurement of pore radius.

The limitation of the model proposed by [1] are obviously due to the simplifying hypotheses and due to retention mechanisms, that were neglected. The model neglects the presence of microcracks, root channels, particle aggregation, and non-uniform packing density. In addition, the fact that the pore radius $\left(r_{i}\right)$ is dependent on the number of particles $\left(n_{i}\right)$ is physically untenable, as pointed out by [2]. Despite its limitations, the model proposed by [1] has established a general approach that was followed by many other authors.

To improve Arya and Paris model, [2] proposed an alternative formulation for pore radii. To remove the number of particles $\left(n_{i}\right)$ from the formulation, the authors proposed the introduction of the parameter a new empirical parameter, $\alpha^{*}$, defining the total pore length ( $h_{i}$ ) as equal to $n_{i} \alpha^{*}$. Thus, Equation 6 becomes:

$$
r_{i}=\sqrt{\frac{4 e R_{i}^{3}}{3 \alpha^{*}}}
$$

For the soils tested, the alternative model presented reasonable agreement between the measured and predicted SWCCs. The authors pointed out that the parameter $\alpha^{*}$ has a narrow range of values. In other words, the modified model proposed by [2] was not as sensitive to the empirical parameter $\alpha^{*}$ when compared to the sensitivity of the model by [1] with respect to $\alpha$.

\subsection{Aubertin et al. [3]}

Basic geotechnical properties of granular and finegrained materials were used by [3] to predict the SWCC, considering capillarity and particle adhesion. The authors presented an expression (Equation 9) relating the degree of saturation and matric suction diving the retention into adhesive and capillary portions:

$$
S=S_{c}+S_{a}^{*}\left(1-S_{c}\right)
$$

where: $S_{c}$ is the degree of saturation associated with capillary forces; and $S_{a}^{*}$ is the degree of saturation caused by adhesion and does not exceed unity at low suctions and defined as:

$$
S_{a}^{*}=1-\left\langle 1-S_{a}\right\rangle
$$


where: \langle\rangle represents the Macauley brackets ( $\langle y\rangle=0.5(y+|y|))$; for $S_{a} \geq 1, S_{a}^{*}=1$, and for $S_{a}<1$, $S_{a}^{*}=S_{a}$.

The degrees of saturation associated with capillary forces (Equation 11) and with adhesion (Equation 12) are express as functions of the equivalent capillary rise in the porous medium $\left(h_{c o}\right)$ and matric suction:

$$
\begin{gathered}
S_{c}=1-\left[\left(\frac{h_{c o}}{u_{a}-u_{w}}\right)^{2}+1\right]^{m} \exp \left[-m\left(\frac{h_{c o}}{u_{a}-u_{w}}\right)^{2}\right] \\
S_{a}=a_{c} C_{\left(u_{a}-u_{w}\right)} \frac{\left(h_{c o} / \psi_{n}\right)^{2 / 3}}{e^{1 / 3}\left(\left(u_{a}-u_{w}\right) / \psi_{n}\right)^{1 / 6}} \\
C_{u_{a}-u_{w}}=1-\frac{\ln \left(1+\frac{u_{a}-u_{w}}{\left(u_{a}-u_{w}\right)_{r}}\right)}{\ln \left(1+\frac{\left(u_{a}-u_{w}\right)_{0}}{\left(u_{a}-u_{w}\right)_{r}}\right)}
\end{gathered}
$$

where: $m$ is identified by the authors as the distribution parameter associated with the influence of the PSD curve; $a_{c}$ is the adhesion coefficient; $\psi_{m}$ is a normalization parameter; $\left(u_{a}-u_{w}\right)_{r}$ is the residual suction; and $\left(u_{a}-u_{w}\right)_{0}$ is the suction at complete dryness.

The residual suction $\left(u_{a}-u_{w}\right)_{r}$ is calculated according to the material. Parameters from the GSD such as $D_{10}$ (diameter corresponding to $10 \%$ passing) and $C_{U}$ (coefficient of uniformity) are used for granular soils while the liquid limit $\left(w_{L}\right)$ is used for plastic-cohesive materials. Parameters $a_{c}$ and $m$ were determined from a fitting procedure using experimental data and the basic properties aforementioned.

The authors obtained good agreement between the predicted and measured curves. It was pointed out that there was no clear correlation between the air-entry value and basic geotechnical properties for plasticcohesive materials. However, for granular materials, the model overestimates of the air-entry value by only about $17 \%$.

Applying the Aubertin et al. [3] model requires caution since some factors such as compaction, hysteresis, volume-change, stress-strain history, multimodal pore size distribution curves and the presence of very coarse particles were not considered. As positive points of the model, the distinction between adhesion and capillary forces can provide more accurate results, mainly for finer soils. In addition, the use of basic geotechnical properties, such as the grain-size distribution curve $\left(D_{10}, C_{U}\right)$, porosity, the density of the particles, and liquid limit $\left(w_{L}\right)$, is an advantage of the method, once it requires only routine laboratory tests to predict the SWCC.

\subsection{Wang et al. [4]}

Using the van Genuchten's water retention model as basis, [4] developed a model to estimate the SWCC of granular soils based on a semi physical and semi statistical approach. The van Genuchten equation was presented in terms of degree of saturation, as follows:

$$
S=\left(1+\left(\frac{u_{a}-u_{w}}{\alpha}\right)^{n}\right)^{1 / n-1}
$$

The authors used dimensional analysis to propose the following equation for the variables $n$ and $\alpha$, which are related to $D_{60}$ and $C_{U}$, respectively:

$$
\begin{gathered}
n=\frac{C_{1}}{\log _{10}\left(C_{U}\right)}+1 \\
\alpha=\frac{C_{2} \gamma}{D_{60}}
\end{gathered}
$$

A database of 78 granular materials were used to establish the parameters $C_{1}$ and $C_{2}$. The obtained values were 1.07 and 12.07 , respectively. The validity of the model was tested by estimating the SWCC of six additional soils from the literature. The results showed good agreements between measured and predicted curves.

\section{Materials}

A dataset of 16 glass beads selected from the literature combined with 3 additional glass beads studies by in the present research project was selected to evaluate the performance of the SWCC prediction models. Table 1 presents the basic information about each material.

Figures 1 and 2 show the grain-size distribution curves of the dataset. Except for the glass beads \#1, all materials present low coefficients of uniformity. Notice that the grain-size distribution curves of materials \#4 to \#11 are defined with only 3 points. In these cases, the GSD is undetermined between 0 and approximately $40 \%$ passing. This is a problem for models that use the entire the grain-size distribution curve instead of a fitting equation, namely Arya and Paris [1] and Arya and Dierolf [2]. As consequence, the predictions using these models is also incomplete.

The diameter of the particles of the various glass beads has a relatively wide range, varying by nearly 100 times. The coefficients of uniformity are between 1.04 to 2.19. It is also important to point out that the dataset offers an opportunity to evaluate the effect of porosity, with identical material evaluated at two porosities. The only difference between materials \#4 and \#5 is the porosity. The same applies to materials \#6 and \#7, \#8 and \#9 and \#10 and \#11.

The tested models do not consider hysteresis effects, they all were developed to predict only the primary drying curves. For this reason, the dataset contains only these types of curves. 
Table 1. Basic properties of the dataset.

\begin{tabular}{|c|c|c|c|c|c|}
\hline$\#$ & Reference & $\begin{array}{c}\boldsymbol{\rho}_{\mathrm{s}} \\
\left(\mathrm{g} / \mathrm{cm}^{3}\right)\end{array}$ & $\begin{array}{c}\rho_{\mathbf{d}} \\
\left(\mathbf{k g} / \mathbf{m}^{3}\right)\end{array}$ & $\mathbf{n}$ & $\mathbf{C}_{\mathbf{U}}$ \\
\hline 1 & \multirow{3}{*}{$\begin{array}{l}\text { Authors' } \\
\text { results }\end{array}$} & 2.49 & 1658 & 0.33 & 2.19 \\
\hline 2 & & 2.46 & 1589 & 0.35 & 1.09 \\
\hline 3 & & 2.49 & 1574 & 0.37 & 1.04 \\
\hline 4 & \multirow{8}{*}{ [9] } & 2.98 & 1922 & 0.36 & \multirow{2}{*}{1.17} \\
\hline 5 & & 2.98 & 1809 & 0.39 & \\
\hline 6 & & 2.98 & 1931 & 0.35 & \multirow{2}{*}{1.06} \\
\hline 7 & & 2.98 & 1788 & 0.40 & \\
\hline 8 & & 2.98 & 1952 & 0.35 & \multirow{2}{*}{1.07} \\
\hline 9 & & 2.98 & 1836 & 0.38 & \\
\hline 10 & & 2.98 & 1946 & 0.35 & \multirow{2}{*}{1.11} \\
\hline 11 & & 2.98 & 1797 & 0.40 & \\
\hline 12 & \multirow{3}{*}{ [10] } & 2.65 & 1656 & 0.38 & 1.14 \\
\hline 13 & & 2.65 & 1606 & 0.39 & 2.22 \\
\hline 14 & & 2.65 & 1532 & 0.42 & 1.42 \\
\hline 15 & [11] & 2.50 & 1785 & 0.29 & 1.23 \\
\hline 16 & \multirow{4}{*}{ [4] } & 2.50 & 1413 & 0.44 & 1.25 \\
\hline 17 & & 2.50 & 1493 & 0.40 & 1.25 \\
\hline 18 & & 2.50 & 1468 & 0.41 & 1.47 \\
\hline 19 & & 2.50 & 1493 & 0.40 & 1.79 \\
\hline
\end{tabular}

\section{Results and discussions}

Figures 3 to 5 present the results of the prediction exercises for three materials. These materials were selected for presentation because they are representative of the entire dataset. Similar results were obtained for the remaining materials.

The Arya and Paris [1] model overestimated suction for all materials, typically by half to one order of magnitude (i.e., log cycle). In terms of the shape of the SWCC, this model seems to provide better results for materials with relatively higher coefficients of uniformity (Figures 3 to 5).

The results from Arya and Dierolf [2] model are quite similar to those from the Arya and Paris [1] model in terms of the SWCC overall shape, but the first offer grater overestimations of matric suction. This model presented the poorest results among all models. The Arya and Dierolf [2] model has the same limitation of its predecessor regarding the need for grain-size distribution curves with a small number of determination points. This limitation could be overcome by using best-fit equations to represent the GSD, such as those proposed by [12].

The model proposed by Aubertin et al. [3] provided superior predictions when compared with the previous models. The model slightly underestimated matric suctions for some materials, with differences lower than 0.2 of one log cycle. In terms of SWCC slope, the model proposed by Aubertin et al. [3] offered inconsistent results. For some materials, it provided excellent predictions with slopes nearly identical. However, for 9 out of the 19 glass beads, the predicted SWCC had a noticeably flatter slope. No relationship was observed in terms of the coefficient of uniformity and these prediction imperfections. These relatively minor inconsistencies may be attributed to the complex particle assemblage, not considered by these prediction models.

The model introduced by Wang et al. [4] also provided superior predictions when compared to the models proposed in [1] and [2]. Matric suction was still slightly overestimated for most materials, but the SWCC slopes are relatively accurate. The superior performance of the model by Wang et al. [4] is in part due the broader dataset on which it was based, that specifically includes glass beads.

According to three statistical parameters from the regression analysis shown in Table 1, the best prediction methods were from those proposed by Aubertin et al. [3] and by Wang et al. [4]. Both models are not based only in particle and pores geometry as they include empirical observations that allowed the consideration of the complexity of the soil-water interactions. The models by Aubertin et al. [3] and Wang et al. [4] models use the coefficient of uniformity of the grain-size distribution curve to control the slope of the SWCC. Consequently, they provided the best results.

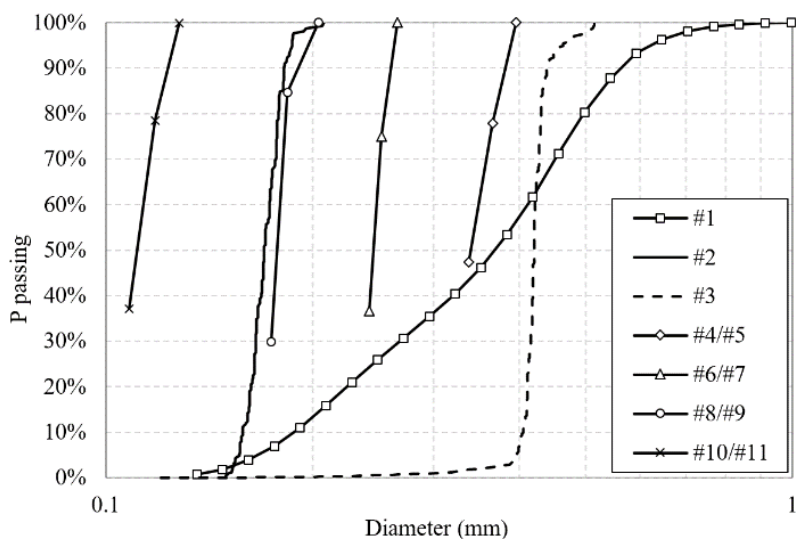

Fig. 1. Grain-size distribution curve of materials \#1 to \#11.

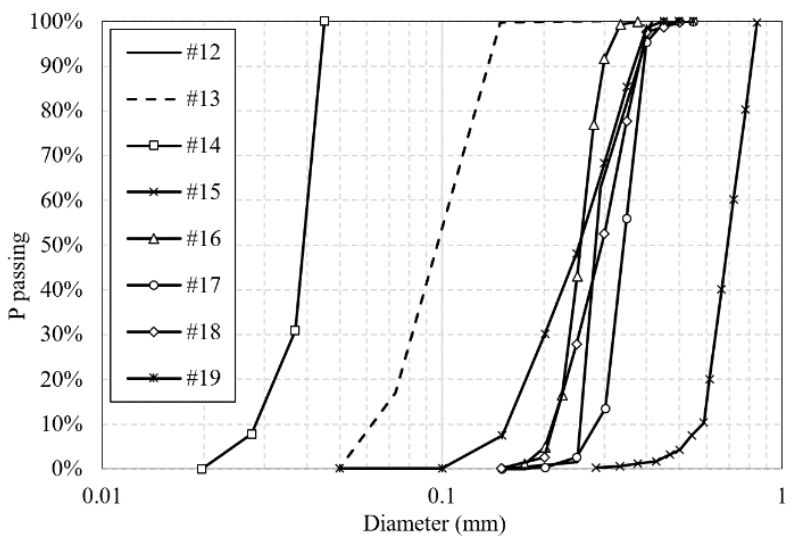

Fig. 2. Grain-size distribution curve of materials \#12 to \#19. 


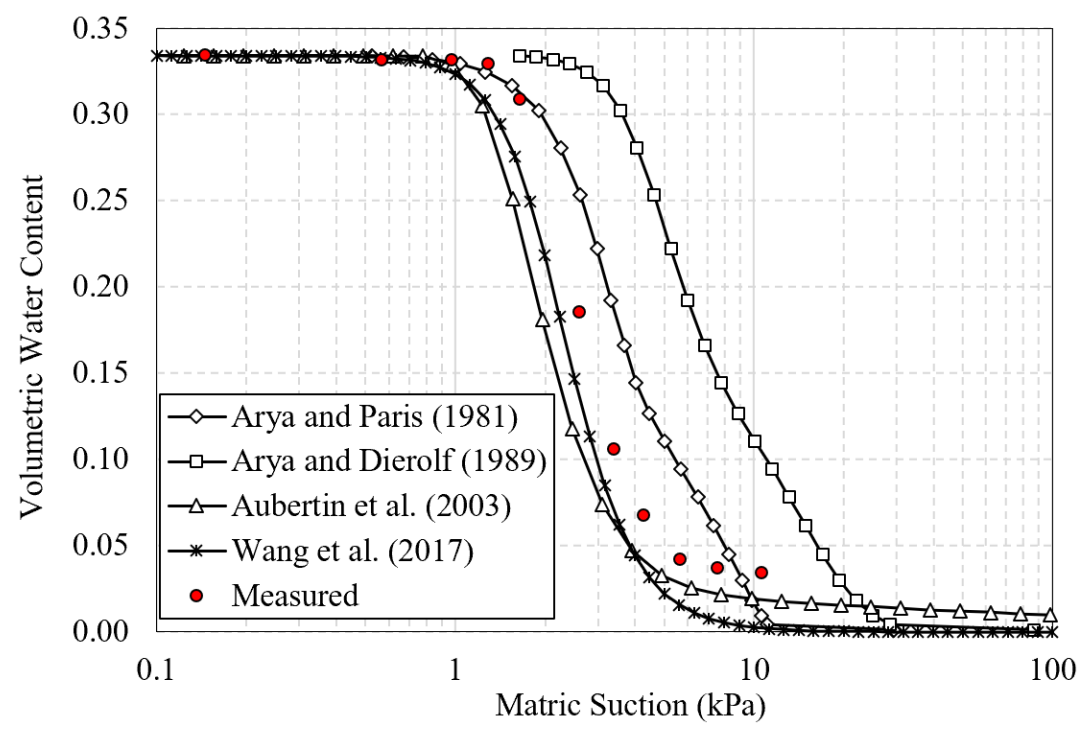

Fig. 3. Measured and predicted SWCC of material \#1.

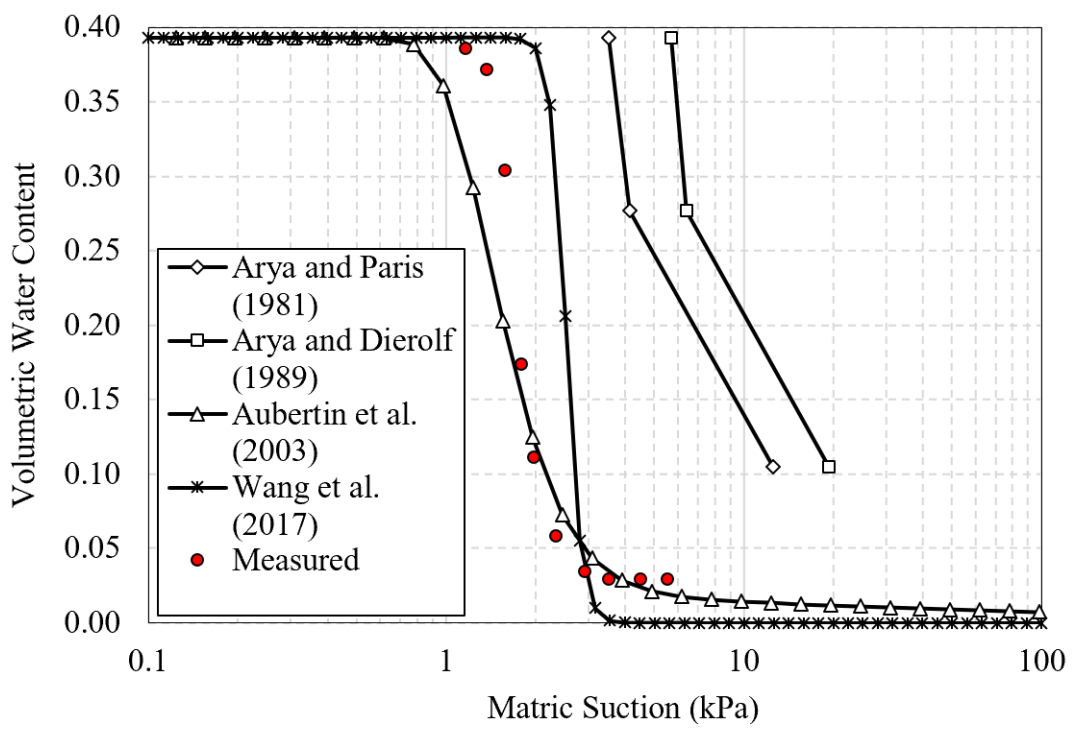

Fig. 4. Measured and predicted SWCC of material \#5.

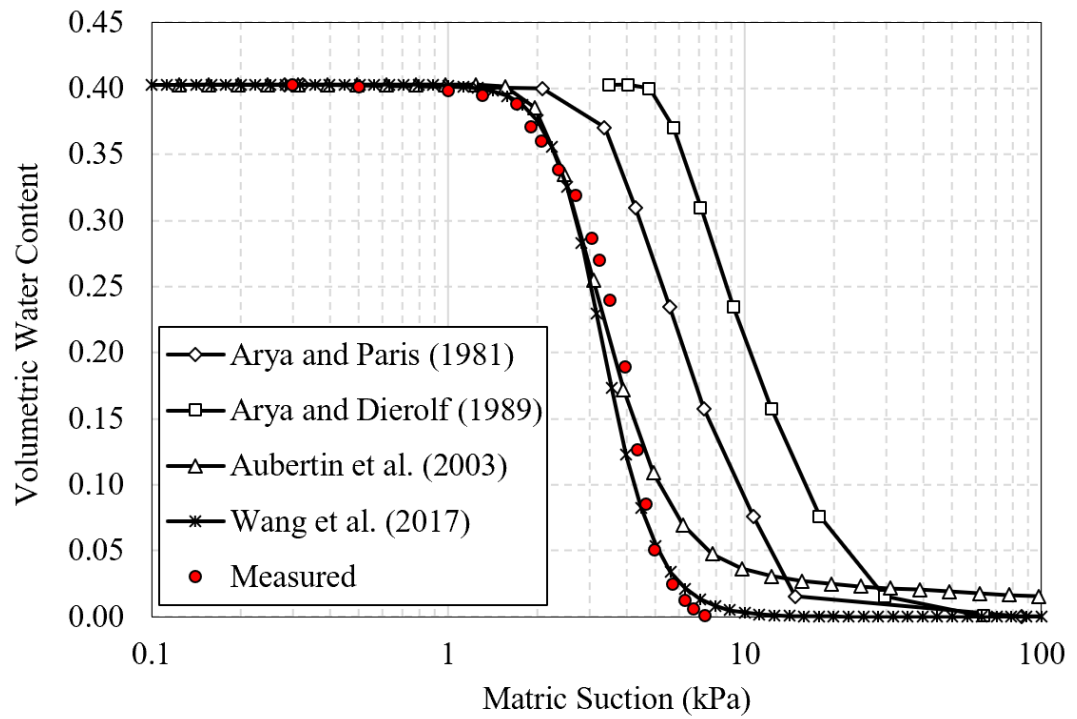

Fig. 5. Measured and predicted SWCC of material \#19. 
Table 2. Average parameters obtained from the regression analysis of the dataset.

\begin{tabular}{|l|c|c|c|}
\hline \multicolumn{1}{|c|}{ Model } & RMSE & SSE & $\mathbf{R}^{\mathbf{2}}$ \\
\hline Arya and Paris [1] & 0.178 & 0.477 & -0.819 \\
\hline Arya and Dierolf [2] & 0.217 & 0.675 & -1.548 \\
\hline Aubertin et al. [3] & 0.071 & 0.075 & 0.698 \\
\hline Wang et al. [4] & 0.077 & 0.092 & 0.567 \\
\hline
\end{tabular}

\section{Conclusions}

Some of the main models available to predict the SWCC are based on simplifications of the particle-pore geometry. Therefore, the investigation of the behaviour of such models using simple ideal materials allows a better understanding of the performance of these models. Glass beads are some of the most simple material that may be used in the calibration and evaluation of prediction models.

Four predictions models, [1 - 4] were evaluated using a dataset comprised of 19 glass beads varying in grain-size distribution, porosity and density of particles. Regression analysis using RMSE, SSE and $\mathrm{R}^{2}$ were performed to measure the goodness of fit of the models.

The Arya and Paris [1] and the Arya and Dierolf [2] models presented similar behaviour of overestimating suction. Despite the fact that the Arya and Dierolf [2] model is supposed to be an improvement of Arya and Paris [1] model, the first earlier original model was superior.

The models that considers the shape of the grain-size distribution curve provided the best results [3 - 4]. Both models were capable of reproducing adequately the airentry value and the slope of the SWCC. The regression analysis indicates the model by Wang et al. [4] is particularly accurate, since it has been developed considering glass beads and sands.

A more comprehensive investigation using sands and considering its irregular particle geometry is necessary to test and the performance of the models and to expand the analyses to a broader dataset.

\section{References}

1. L. M. Arya, J. F. Paris, Soil Sci. Soc. Am. Journal, 45, 1023, (1981)

2. L. M. Arya, T. S. Dierolf, Int. Workshop, 115, (1989)

3. M. Aubertin, M. Mbonimpa, B. Bussière, R. P. Chapuis, Can. Geo. Journal, 40, 1104 (2003)

4. J. Wang, N. Hu, B. François, P. Lambert, Water es. Resech., 53, 6069 (2017)

5. R. Haverkamp, J. Y. Parlange, Soil Sci, 142, 325 (1986).

6. L. M. Arya, F. J. Leij, M. T. van Genuchten, P. J. Shouse, Soil Sci. Soc. Am. Journal, 63, 510 (1999)

7. M. D. Fredlund, G. W. Wilson, D. G. Fredlund, Can. Geo. Journal, 39, 1103 (2002)

8. W. J. Likos, R. Jaafar, Journal Geotech. And Geoenv. Eng, 139, 724 (2013)

9. J. A. Dodds, P. Srivastava, Particle \& particles systems characterization, 23, 29 (2006)

10. R. Jaafar, W. J. Likos, Geo. Test. Journal, 34, 1 (2011)

11. K. N. Manahiloh, B. Muhunthan, W. J. Likos, Int. Journal Geomech., 16, 1 (2016)

12. M. D. Fredlund, D. G. Fredlund, G. W. Wilson, Can. Geo. Journal, 37, 817 (2000) 